VoL. 51 (1995) [479-488]

\title{
A RIGHT CONTINUOUS RIGHT WEAKLY SI-RING IS SEMISIMPLE
}

\section{Dinh van HuYnh and NguYen van Sanh}

\begin{abstract}
It is shown that a projective CS right module $M$ over a ring $R$ is a direct sum of uniform modules of composition lengths at most 2 if (i) every finitely generated direct summand of $M$ is continuous and (ii) every non-zero $M$-singular right $R$-module contains a non-zero $M$-injective submodule. In particular, a right continuous ring $R$ is semisimple if $R$ is right weakly SI, that is, if every non-zero singular right $R$-module contains a non-zero injective submodule.
\end{abstract}

\section{INTRODUCTION}

Right (left) SI-rings, that is, rings all of whose singular right (left) modules are injective, were introduced and investigated in detail by Goodearl [9]. Since then SIrings have drawn much attention from several authors, see for example, $[7,10,13,14$, $15,16,17,20]$. In a similar way, SI-modules have been defined and considered in [20] and $[10]$ where corresponding properties were obtained.

A weaker form of SI-rings and SI-modules was considered recently in [15]: A right $R$-module $M$ is called weakly SI (briefly, WSI) if every non-zero $M$-singular right $R$ module contains a non-zero $M$-injective submodule. A ring $R$ is called a right WSI-ring if $R_{R}$ is WSI.

As shown in [15], WSI-modules have some properties similar to those of SI-modules. However, in general the structure of them still remains unknown. It is clear that any right semiartinian right $\mathrm{V}$-ring is right WSI. By [2], [3, Theorem 2.2] or [6, Corollary 21] there exists a right semiartinian right $V$-ring $R$ such that the right Loewy series of $R$ has (Loewy) length at least 3. By [9, Theorem 3.11] such a ring $R$ is not right SI. Hence right WSI-rings are not necessarily right SI, in general. On the other hand, a right PCI-domain constructed in [5] is right SI, and so it is right WSI but not right semiartinian. This means that right WSI-rings need not be right semiartinian. Therefore WSI-modules and WSI-rings seem to be interesting subjects in the area.

The purpose of this note is to prove the following results. For the definition of the category $\sigma[M]$ we refer to the next section.

Received 1st August, 1994

Copyright Clearance Centre, Inc. Serial-fee code: 0004-9729/95 \$A2.00+0.00. 
Theorem 1. Let $M$ be a WSI right $R$-module which is projective in $\sigma[M]$. Then $M$ is $M$-nonsingular. Assume furthermore that $M$ is $C S$, then

(a) $M$ is a direct sum of finitely generated modules $M_{i}$, where each $M_{i}$ has either zero socle or $M_{i}$ is a semiartinian module and $\bar{M}_{i}=M_{i} / \operatorname{Soc}\left(M_{i}\right)$ is a $V$-module, that is, every simple module in $\sigma\left[\bar{M}_{i}\right]$ is $\bar{M}_{i}$-injective.

(b) If every finitely generated direct summand of $M$ is quasi-continuous, then $M$ is quasi-continuous and $M=\underset{i \in \Omega}{\oplus} U_{i}$ where each $U_{i}$ is a finitely generated uniform submodule of $M$. Moreover, if $\operatorname{Soc}\left(U_{i}\right) \neq 0$ for some $i \in \Omega$, then $U_{i}$ has composition length $\leqslant 2$, and each $U_{j}$ with $S o c\left(U_{j}\right)=0$ is a fully invariant submodule of $M$. Therefore, in this case, $M$ is a direct sum of a fully invariant SI-submodule with essential socle and fully invariant uniform submodules with zero socles.

(c) If every finitely generated direct summand of $M$ is continuous, then $M$ is a continuous $S I$-module which is a direct sum of uniform submodules with composition lengths $\leqslant 2$. Moreover, in this case, if $N_{R}$ is a finitely generated direct summand of $M$, then $\operatorname{End}_{R}(N)$ is a semisimple ring.

The following consequence of Theorem 1 improves [6, Lemma 10] which stated that a right self-injective ring $R$ is semisimple if every non-zero right $R$-module contains a non-zero injective submodule.

Corollary 2. Any right continuous right WSI-ring is semisimple.

For quasi-continuous rings we have:

Corollary 3. Every right quasi-continuous right WSI-ring is the ring direct sum of a semisimple ring and finitely many right Ore domains which are not division rings. In particular, any right quasi-continuous, right semiartinian right $V$-ring is semisimple.

The last statement of Corollary 3 gives the possibility of producing several von Neumann regular right $\mathrm{V}$-rings with zero right socle.

The following result is an easy consequence of (a) in Theorem 1.

Corollary 4. A right $C S$ right WSI-ring $R$ has a ring direct decomposition $R=A \oplus B$ where $A$ is a right semiartinian ring such that $A / \operatorname{Soc}\left(A_{A}\right)$ is a right $V$-ring and $\operatorname{Soc}\left(B_{B}\right)=0$.

\section{Preliminaries}

Throughout this note all rings are associative with identity and all modules are unitary modules.

For a module $M$ over a ring $R$ we write $M_{R}$ to indicate that $M$ is a right $R$ module. The socle and the Jacobson radical of $M$ are denoted respectively by $S o c(M)$ 
and $J(M)$. A module $M$ is called semisimple if $M=S o c(M)$, and a ring $R$ is said to be a semisimple ring if $R_{R}$ is semisimple, or equivalently, if $R$ is a semiprime right (or left) Artinian ring. A submodule $N$ of a module $M_{R}$ is called a fully invariant submodule of $M$ if for each $f \in \operatorname{End}_{R}(M), f(N) \subseteq N$.

For a given module $M_{R}$ we consider the following properties:

$\left(C_{1}\right)$ Every submodule of $M$ is contained essentially in a direct summand of $M$.

$\left(C_{2}\right)$ If $A$ and $B$ are direct summands of $M$ with $A \cap B=0$, then $A \oplus B$ is also a direct summand of $M$.

$\left(C_{3}\right)$ If $C$ is a submodule of $M$ isomorphic to a direct summand of $M$, then $C$ is itself a direct summand of $M$.

A module $M_{R}$ is said to be a CS-module if it satisfies $\left(C_{1}\right) ; M$ is called quasicontinuous if $M$ satisfies $\left(C_{1}\right)$ and $\left(C_{2}\right)$ and finally, if $M$ satisfies $\left(C_{1}\right)$ and $\left(C_{3}\right)$ then $M$ is said to be a continuous module. We have the following implications:

injective $\Rightarrow$ quasi-injective $\Rightarrow$ continuous $\Rightarrow$ quasi-continuous $\Rightarrow$ CS.

In general these classes are distinct.

A ring $R$ is called right CS (right quasi-continuous, right continuous) if $R_{R}$ is CS (quasi-continuous, continuous). For a detailed study of these classes of rings and modules we refer to Dung-Huynh-Smith-Wisbauer [7] and Mohamed-Müller [11].

For a module $M_{R}$ (over a ring $R$ ) we denote by $\sigma[M]$ the full subcategory of Mod$R$ (the category of all right $R$-modules) whose objects are submodules of $M$-generated modules (see Wisbauer [19]). A module $P \in \sigma[M]$ is called projective in $\sigma[M]$ if $P$ is $N$-projective for every $N \in \sigma[M]$. A module $U_{R}$ is called $M$-singular if there is a module $A \in \sigma[M]$ containing an essential submodule $E$ such that $U \simeq A / E$. Hence any $M$-singular module is contained in $\sigma[M]$. For $M=R$ the notion of $R$-singularity is identical to the usual definition of singular $R$-modules in Mod- $R$ (see Goodearl [9]).

The class of $M$-singular right $R$-modules is closed under taking submodules, homomorphic images and direct sums (for example, $[19,17.3,17.4])$. Hence any $N \in \sigma[M]$ contains a largest $M$-singular submodule which is denoted by $Z_{M}(N)$. If $Z_{M}(N)=0$, then $N$ is called $M$-nonsingular.

A module $M$ is called an SI-module if every $M$-singular module is $M$-injective, and $M$ is called weakly SI (briefly WSI) if every non-zero $M$-singular module contains a non-zero $M$-injective submodule. Clearly, any SI-module is WSI. However, in general the converse is not true, as mentioned in the Introduction. A ring $R$ is right WSI if $R_{R}$ is a WSI-module.

The texts by Anderson - Fuller [1], Chatters - Hajarnavis [4], Faith [8], Goodearl [9], Mohamed - Müller [11], Stenström [18] and Wisbauer [19] are general references for module and ring theoretic notions not defined in this note. 


\section{The Proofs}

The following special case of a recent result of Osofsky [12, Theorem B] is the key lemma of our proof of Theorem 1.

Lемма 4. Let $M_{R}$ be a finitely generated, quasi-continuous and quasi-projective module such that no non-zero element of $\operatorname{End}_{R}(M)$ has essential kernel. Then for each set $\left\{e_{i}\right\}_{i=1}^{\infty}$ of orthogonal idempotents $e_{i}$ in $\operatorname{End}_{R}(M)$ with $\underset{i=1}{\oplus} e_{i} M$ essential in $M$, the factor module $M /\left(\bigoplus_{i=1}^{\infty} e_{i} M\right)$ cannot contain a non-zero quasi-continuous direct summand.

Proof of Theorem 1: Let $M_{R}$ be a WSI-module such that $M$ is projective in $\sigma[M]$, where $R$ is a ring.

If $Z_{M}(M) \neq 0$, then $Z_{M}(M)$ contains a non-zero $M$-injective submodule $N$. Hence $N$ is a direct summand of $M$, and therefore $N$ is projective in $\sigma[M]$, a contradiction. Thus $Z_{M}(M)=0$, that is, $M$ is $M$-nonsingular.

From now on we assume in addition that $M$ is a CS-module.

Claim 1. Any finitely generated submodule $U$ of $M$ is essential in a finitely generated direct summand of $M$.

In fact this claim can be derived from [7, Proposition 2.7], however we give a proof here (with a similar argument) for the sake of completeness. Since $M$ is CS, there is a direct summand $U^{*}$ of $M$ such that $U$ is essential in $U^{*}$. (We assume $U \neq 0$, since for $U=0$ the statement is clear). To verify Claim 1 we shall show that $U_{R}^{*}$ is finitely generated.

Clearly, $U^{*}$ is projective in $\sigma[M]$ and $M$-nonsingular. Let $\left\{x_{\lambda}, \lambda \in \Lambda\right\}$ be a generating set of $U^{*}$, that is,

$$
U^{*}=\sum_{\lambda \in \Lambda} x_{\lambda} R
$$

Then there exists an epimorphism $g$ from $\underset{\lambda \in \Lambda}{\oplus} x_{\lambda} R$ onto $U_{R}^{*}$. Since $U^{*}$ is projective in $\sigma[M]$ and $\underset{\lambda \in \Lambda}{\oplus} x_{\lambda} R \in \sigma[M]$, the map $g$ splits (see $[19,18.3]$ ), that is, there exists a submodule $H$ of $\underset{\lambda \in \Lambda}{\oplus} x_{\lambda} R$ with $H \simeq U^{*}$ and

$$
\underset{\lambda \in \Lambda}{\oplus} x_{\lambda} R=H \oplus K \operatorname{er}(g)
$$

It is clear that $H$ also contains a finitely generated essential submodule $K$ (since $\left.H \simeq U^{*}\right)$. Let $K=y_{1} R+\ldots+y_{n} R\left(y_{i} \in H\right)$ and for each $\mu \in \Lambda$ let $e_{\mu}$ be the canonical projection of $\underset{\lambda \in \Lambda}{\oplus} x_{\lambda} R$ onto $x_{\mu} R$. Since for each $x \in H, e_{\mu}(x) \neq 0$ only for finitely many $\mu \in \Lambda$, the set

$$
\Gamma=\left\{\gamma \in \Lambda, e_{\gamma}\left(y_{i}\right) \neq 0 \text { for some } y_{i}, 1 \leqslant i \leqslant n\right\}
$$


is finite. Hence for every $\lambda \in \Lambda \backslash \Gamma, e_{\lambda}(K)=0$. This shows that $K$ is contained in $\operatorname{Ker}\left(e_{\lambda}\right)$ for each $\lambda \in \Lambda \backslash \Gamma$. Therefore for such $\lambda, e_{\lambda}(H)$ is an $M$-singular submodule of $x_{\lambda} R$. But $x_{\lambda} R \subseteq M$ and $M$ is $M$-nonsingular. It follows that $e_{\lambda}(H)=0$ for all $\lambda \in \Lambda \backslash \Gamma$. Hence

$$
H \subseteq \underset{\gamma \in \Gamma}{\oplus} x_{\gamma} R .
$$

This together with (1) shows that $H$ is a direct summand of $\underset{\gamma \in \Gamma}{\oplus} x_{\gamma} R$, and so $H_{R}$ is finitely generated. Thus $U_{R}^{*}$ is finitely generated, as desired.

(a) Since $M$ is projective in $\sigma[M]$, by Kaplansky's Theorem (see $[19,8.10,18.4]$ ) $M$ is a direct sum of countably generated modules. Hence to prove (a), we may assume that $M$ is countably generated, say

$$
M=\sum_{i=1}^{\infty} x_{i} R .
$$

Note that $M$ is $M$-nonsingular as shown before Claim 1. Now let $M_{1}$ be a maximal essential extension of $x_{1} R$ in $M$. By hypothesis we have

$$
M=M_{1} \oplus M_{1}^{\prime} .
$$

By Claim 1, $M_{1}$ is finitely generated. Assume inductively that for some positive integer $n \geqslant 1$, we already found finitely many independent submodules $M_{1}, \ldots, M_{n}$ each of which is finitely generated and

$$
M=\left(M_{1} \oplus \cdots \oplus M_{n}\right) \oplus M_{n}^{\prime}
$$

such that $x_{1} R+\cdots+x_{n} R \subseteq M_{1} \oplus \cdots \oplus M_{n}$. Let $\pi$ be the projection of $M$ onto $M_{n}^{\prime}$ and let $x_{n+1}^{\prime}=\pi\left(x_{n+1}\right)$. Since $M_{n}^{\prime}$ is also a CS and WSI-module which is projective in $\sigma[M]$, we may use the first step above to find a finitely generated direct summand $M_{n+1}$ of $M_{n}^{\prime}$ such that $x_{n+1}^{\prime} R$ is essential in $M_{n+1}$. Thus

$$
M=M_{1} \oplus \cdots \oplus M_{n+1} \oplus M_{n+1}^{\prime}
$$

with $x_{1} R+\cdots+x_{n+1} R \subseteq M_{1} \oplus \cdots \oplus M_{n+1}$. This induction argument shows that $M$ contains an independent set $\left\{M_{i}\right\}_{i=1}^{\infty}$ of finitely generated submodules such that $\sum_{i=1}^{\infty} x_{i} R \subseteq \underset{i=1}{\oplus} M_{i}$. Therefore $M=\underset{i=1}{\oplus} M_{i}$ as desired.

Put $M=\underset{i \in I}{\oplus_{i}} M_{i}$ where each $M_{i}$ is finitely generated and let $S_{i}$ be the socle of $M_{i}$. Assume that for some $i \in I, S_{i} \neq 0$. Since $M$ is CS we have $M_{i}=A_{i} \oplus B_{i}$ where $S o c\left(B_{i}\right)=0$ and $S_{i}$ is essential in $A_{i}$. Hence $\bar{A}_{i}=A_{i} / S_{i}$ is an $M$-singular module. Therefore each non-zero subfactor of $\bar{A}_{i}$ contains a non-zero $M$-injective submodule. Moreover, since $S_{i}$ is a fully invariant submodule of $A_{i}, \bar{A}_{i}$ is quasi-projective. Hence we may use the main result of [6] to see that $\bar{A}_{i}$ is a semiartinian V-module, and it follows that $A_{i}$ is semiartinian. This fact verifies (a).

(b) Assume that every finitely generated direct summand of $M$ is quasi-continuous. 
Claim 2. Any finitely generated submodule of $M$ has finite uniform dimension.

Let $V$ be a finitely generated submodule of $M$. Then $V$ is an essential submodule of a finitely generated direct summand $V^{*}$ of $M$ by Claim 1 . Assume on the contrary that $V$ does not have finite uniform dimension. Then $V$ contains an infinite independent set $\left\{V_{i}\right\}_{i=1}^{\infty}$ of non-zero submodules $V_{i}$. Put $W=\bigoplus_{i=1}^{\infty} V_{i}$. Since $V^{*}$ is also a CS-module, $W$ is contained as an essential submodule in a direct summand $W^{*}$ of $V^{*}$. Clearly $W^{*}$ is finitely generated and a direct summand of $M$. Hence $W^{*}$ is quasi-continuous. Moreover $W^{*}$ is projective in $\sigma[M]$.

Let $V_{i}^{*}$ be a maximal essential extension of $V_{i}$ in $W^{*}$ for each $i=1,2, \ldots$. Then, since $W^{*}$ is quasi-continuous, any finite direct sum $\underset{i=1}{\oplus} V_{i}^{*}$ is a direct summand of $W^{*}$. Let $e_{i}$ be the canonical projection of $W^{*}$ onto $V_{i}^{*}$. Then $\left\{e_{i}\right\}_{i=1}^{\infty}$ is a family of orthogonal idempotents in $S=\operatorname{End}_{R}\left(W^{*}\right)$ with $e_{i} W^{*}=V_{i}^{*}$ and so $\underset{i=1}{\oplus} e_{i} W^{*}$ is essential in $W^{*}$. Note that $\oplus_{i=1}^{\infty} e_{i} W^{*} \neq W^{*}$, since $W^{*}$ is finitely generated, and that for each $0 \neq f \in S, \operatorname{Ker}(f)$ is not essential in $W^{*}$ since $W^{*}$ is $M$-nonsingular. Now we may apply Lemma 4 to see that the non-zero $M$-singular module $W^{*} /\left(\underset{i=1}{\oplus} e_{i} W^{*}\right)$ does not contain a non-zero $M$-injective submodule. However this is a contradiction to the assumption that $M$ is WSI. Thus $V$ must have finite uniform dimension, proving Claim 2.

Now by (a) $M$ is a direct sum of finitely generated modules. Then from the assumption of (b) and Claim 2 it is easy to derive a decomposition of $M$ as a direct sum of finitely generated uniform modules $U_{i}$ :

$$
M=\bigoplus_{i \in \Omega} U_{i}
$$

Next we show that $M$ is quasi-continuous. By [11, Theorem 2.13], it is enough to show that each $M(\Omega \backslash i)$ in (2) is $U_{i}$-injective, where $M(\Omega \backslash i)=\underset{j \in \Omega \backslash i}{\oplus} U_{j}$. Let $V$ be a submodule of $U_{i}$ and $g$ be a non-zero homomorphism of $V$ to $M(\Omega \backslash i)$. Since $M(\Omega \backslash i)$ is $M$-nonsingular, $K \operatorname{er}(g)$ must be zero, that is, $V \simeq g(V)$, in particular $g(V)$ is a uniform submodule of $M(\Omega \backslash i)$. Since $M$ is $M$-nonsingular, the closure of any uniform submodule $H$ in $M$ equals the closure of any non-zero cyclic submodule of $H$. Hence we may use Claim 1 to see that $g(V)$ is an essential submodule of a finitely generated direct summand $W$ of $M(\Omega \backslash i)$. We have $M(\Omega \backslash i)=W \oplus M^{\prime}$ for some submodule $M^{\prime}$ of $M(\Omega \backslash i)$. Hence $U_{i} \oplus W$ is a finitely generated direct summand of $M$. By assumption, $U_{i} \oplus W$ is quasi-continuous and so $W$ is $U_{i}$-injective by [11, Corollary 2.14]. Since $g(V) \subseteq W$, it follows that $g$ can be extended to a homomorphism from $U_{i}$ to $W$. This implies the $U_{i}$-injectivity of $M(\Omega \backslash i)$. 
Assume now that in (2) there is a $U_{j}(j \in \Omega)$ such that $\operatorname{Soc}\left(U_{j}\right) \neq 0$. If $U_{j}$ is simple then we are done. Assume that $U_{j}$ is not simple. Let $S_{j}=\operatorname{End}_{R}\left(U_{j}\right)$. If there is a non-zero element $f \in S_{j}$ such that $f\left(U_{j}\right)$ is small in $U_{j}$ then $f\left(U_{j}\right)$ is contained in each maximal submodule of $U_{j}$, that is, $f\left(U_{j}\right) \subseteq J\left(U_{j}\right)$. Since $S o c\left(U_{j}\right)$ is contained in each non-zero submodule of $U_{j}$, we also have $\operatorname{Soc}\left(U_{j}\right) \subseteq J\left(U_{j}\right)$. On the other hand, since $U_{j}$ is a finitely generated quasi-projective WSI-module, $J\left(U_{j}\right) \subseteq S o c\left(U_{j}\right)$ by $[15$, Proposition 4]. Hence $J\left(U_{j}\right)=S o c\left(U_{j}\right)$. It follows that $f\left(U_{j}\right)=S o c\left(U_{j}\right)$. From this we must have $\operatorname{Ker}(f) \neq 0$, since $U_{j}$ is not simple. This implies that $f\left(U_{j}\right)$ is $M$-singular, a contradiction. Thus for each $0 \neq f \in S_{j}, f\left(U_{j}\right)$ is not small in $U_{j}$. By $[19,22.2]$ we have $J\left(S_{j}\right)=0$, and

$$
S_{j} \simeq \operatorname{End}_{R}\left(U_{j} / J\left(U_{j}\right)\right)
$$

Furthermore, since each non-zero element of $S_{j}$ must have zero kernel, $S_{j}$ is a domain, in particular $S_{j}$ has only one non-zero idempotent. Hence by (3) we see that $U_{j} / J\left(U_{j}\right)$ is indecomposable. On the other hand, any non-zero submodule of the $M$-singular module $U_{j} / J\left(U_{j}\right)$ contains a non-zero $U_{j}$-injective submodule. Thus $U_{j} / J\left(U_{j}\right)$ has to be simple. Since $J\left(U_{j}\right)\left(=S o c\left(U_{j}\right)\right)$ is a minimal submodule of $U_{j}$, $U_{j}$ has composition length 2, proving the first statement in the second part of (b).

To prove the next assertion of (b) we write (2) in the form

$$
M=\left(\underset{\alpha \in \Omega_{1}}{\oplus} U_{\alpha}\right) \oplus\left(\underset{\beta \in \Omega_{2}}{\oplus} U_{\beta}\right)
$$

where any $U_{\alpha}$ and $U_{\beta}$ are uniform (finitely generated) and $\operatorname{Soc}\left(U_{\alpha}\right) \neq 0, \operatorname{Soc}\left(U_{\beta}\right)=0$ $\left(\alpha \in \Omega_{1}, \beta \in \Omega_{2}\right)$. Clearly, $\underset{\alpha \in \Omega_{1}}{\oplus} U_{\alpha}$ and $\underset{\beta \in \Omega_{2}}{\oplus} U_{\beta}$ are fully invariant submodules of $M$. Therefore to end this part we need only to show that each $U_{\beta}$ is a fully invariant submodule of $U=\underset{\beta \in \Omega_{2}}{\oplus} U_{\beta}$. Since $U$ is $M$-nonsingular, it is easy to see that for any $0 \neq f \in \operatorname{End}_{R}(U), f\left(U_{\beta}\right) \subseteq U_{\beta}$ or $f\left(U_{\beta}\right) \cap U_{\beta}=0$. Now assume that $f\left(U_{\beta}\right) \cap U_{\beta}=0$ for some $\beta \in \Omega_{2}$. Since $\left(f \mid U_{\beta}\right)$ is a monomorphism, $f\left(U_{\beta}\right) \simeq U_{\beta}$, and since $M$ is $M$-nonsingular we may use Claim 1 (as explained above) to see that the uniform submodule $f\left(U_{\beta}\right)$ is an essential submodule of a finitely generated direct summand $V$ of $\underset{\gamma \in \Omega_{2} \backslash \beta}{\oplus} U_{\gamma}$. Hence $U_{\beta} \oplus V$ is a direct summand of $M$, and so $U_{\beta} \oplus V$ is quasicontinuous. Therefore $U_{\beta}$ is $V$-injective; consequently, $U_{\beta}$ is $f\left(U_{\beta}\right)$-injective. It follows that $U_{\beta}$ is quasi-injective.

On the other hand, since $U_{\beta}$ is a finitely generated quasi-projective WSI-module with zero socle, each simple module $E$ in $\sigma\left[U_{\beta}\right]$ is $U_{\beta}$-injective [15, Proposition 4]. Hence each such $E$ is $U_{\beta}$-generated and so $U_{\beta}$ is a generator of $\sigma\left[U_{\beta}\right]$ by $[19,18.5]$. Therefore $\sigma\left[U_{\beta}\right]$ is Morita-equivalent to $\operatorname{Mod}-T$ where $T=\operatorname{End}_{R}\left(U_{\beta}\right)$ (see $[19,46.2]$ ). 
Note that $T$ is a domain. Since $U_{\beta}$ is quasi-injective, $T$ is a right self-injective domain. It follows that $T$ is a division ring. Then by the above Morita-equivalence, $U_{\beta}$ must be simple, a contradiction. Thus we only have $f\left(U_{\beta}\right) \subseteq U_{\beta}$, proving the fact that each $U_{\beta}$ is a fully invariant submodule of $M$.

By a standard argument we see that $\underset{\alpha \in \Omega_{1}}{\oplus} U_{\alpha}$ is an SI-module and therefore the proof of (b) is complete.

(c) We assume now that each finitely generated direct summand of $M$ is continuous. It follows that $M$ has a decomposition of the form (2) such that each $U_{i}$ is continuous. By (b), $M$ is quasi-continuous. Hence by [11, Theorem 3.16], $M$ is continuous.

To finish the first statement of $(c)$ it is enough to show that $\operatorname{Soc}\left(U_{i}\right) \neq 0$ for each $i \in \Omega$. Suppose there is a $U_{i}(i \in \Omega)$ with $\operatorname{Soc}\left(U_{i}\right)=0$, and let $S_{i}=\operatorname{End}_{R}\left(U_{i}\right)$. By the argument in the proof of (b), $\sigma\left[U_{i}\right]$ is Morita - equivalent to Mod- $S_{i}$. On the other hand, since $U_{i}$ is uniform, continuous and $M$-nonsingular, for each $0 \neq f \in S_{i}$, $\operatorname{Ker}(f)=0$ and $f\left(U_{i}\right)=U_{i}$, that is, $f$ is an isomorphism, proving that $S_{i}$ is a division ring. By the previous Morita-equivalence, $U_{i}$ must be a simple module, a contradiction to $\operatorname{Soc}\left(U_{i}\right)=0$. Thus each $U_{i}(i \in \Omega)$ has non-zero socle as desired. By (b), $M$ is an SI-module.

Now assume that $N$ is a finitely generated direct summand of $M$. By Claim 2, $N$ has finite uniform dimension. Moreover, since $N$ is then continuous and $M$-nonsingular, it is easy to see that for each $f \in \operatorname{End}_{R}(N), \operatorname{Ker}(f)$ and $\operatorname{Im}(f)$ are direct summands of $N$. Therefore $\operatorname{End}_{R}(N)$ is a (von Neumann) regular ring. Since $N$ has finite uniform dimension, $\operatorname{End}_{R}(N)$ cannot have an infinite set of orthogonal idempotents. Thus $\operatorname{End}_{R}(N)$ is a semisimple ring.

The statement of Corollary 2 follows directly from (c) of Theorem 1.

Proof of Corollary 3: Let $R$ be a right quasi-continuous right WSI-ring. By Theorem 1 we have a ring direct decomposition:

$$
R=A \oplus B
$$

where $A$ is a direct sum of finitely many uniform right ideals with composition lengths at most 2 and $B$ is a direct sum of finitely many fully invariant uniform right ideals $B_{i}$ of $R$ with zero socles. It follows that $B$ is a direct sum of right Ore domains. Now express $A$ in the form: $A=A_{1} \oplus \ldots \oplus A_{n}$ where each $A_{i}$ is uniform and of composition length $\leqslant 2$. Assume that, for example, $A_{1}$ is not simple and let $S_{1}$ be the minimal submodule of $A_{1}$. Since $S_{1}$ is projective (see [15, Corollary 5]) and cyclic, there is a minimal right ideal $T$ of $A$ with $T \simeq S_{1}$ and $A=T \oplus P$ for some right ideal $P$ of $A$. Since $A$ is right quasi-continuous, $T$ is $P$-injective (see [11, Corollary 2.14]). Moreover, since $T \cap A_{1}=0, A_{1}$ is embedded in $P$, and so $T$ is $A_{1}$-injective. But $T \simeq S_{1}$, and 
so $S_{1}$ is $A_{1}$-injective, hence $S_{1}$ is a direct summand of $A_{1}$, a contradiction. Thus $A$ is a semisimple ring.

Concerning (a) of Theorem 1 we would like to ask the question:

$\left(Q_{1}\right)$ Is a finitely generated CS, quasi-projective WSI-module a direct sum of uniform modules?

If the answer for $\left(Q_{1}\right)$ is yes, it follows that a right CS right WSI-ring is the ring direct sum of a right Artinian ring and a semiprime right Goldie ring. Indeed, let $R$ be a right CS right WSI-ring such that $R_{R}$ has finite uniform dimension. Then by Corollary $4, R$ has a ring direct sum $R=A \oplus B$ where $A$ is semiartinian and $\operatorname{Soc}\left(B_{B}\right)=0$. Since $R_{R}$ has finite uniform dimension, $\operatorname{Soc}\left(A_{A}\right)$ is finitely generated, and so by the argument for proving (b) of Theorem 1 we easily see that $A$ is a direct sum of finitely many uniform right ideals of composition lengths at most 2 ; in particular $A$ is right Artinian. Since $B$ is also right WSI, $B$ is right nonsingular (see [15]). From this and since $B_{B}$ has finite uniform dimension, the maximal right quotient ring $Q_{\max }$ of $B$ is semisimple by [18, Theorem XII.2.5]. Then by [18, Proposition XV.3.3] and since $J(B) \subseteq \operatorname{Soc}\left(B_{B}\right)=0$ (see [15]), $Q_{\max }$ is also the classical right quotient ring of $B$. Hence $B$ is a semiprime right Goldie ring.

We should note also that if the answer of $\left(Q_{1}\right)$ were yes, we would have the interesting consequence that any right $\mathrm{CS}$, right semiartinian right $\mathrm{V}$-ring is semisimple. From this surprising conclusion we have the feeling that the answer to $\left(Q_{1}\right)$ might be no.

Furthermore, to our knowledge, it is unknown whether or not a ring as in Corollary 3 is right Noetherian. More generally we would like to ask the question:

$\left(Q_{2}\right)$ Is a semiprime right Goldie right WSI-ring necessarily right Noetherian?

It is clear that any right WSI-ring with right Krull dimension is right Noetherian right SI. If we add to (a) of Theorem 1 the condition that $M / J(M)$ is an SI-module, then by the arguments presented above we obtain that $M$ is SI and it is a direct sum of Noetherian uniform modules each of which is of composition length $\leqslant 2$ or of zero socle. For $M=R$ we get the fact that a right CS right WSI-ring is right Noetherian (and right SI) if and only if $R / J(R)$ is right SI.

\section{References}

[1] F.W. Anderson and K.R. Fuller, Rings and categories of modules (Springer-Verlag, Berlin, Heidelberg, New York, 1974).

[2] G. Baccella, 'Semiartinian V-rings and semiartinian von Neumann regular rings', J. Algebra (to appear).

[3] V. Camillo and K.R. Fuller, 'On Loewy length of rings', Pacific J.Math. 53 (1974), 347-354. 
[4] A.W. Chatters and Hajarnavis, Rings with chain conditions (Pitman, London, 1980).

[5] J. Cozzens and C. Faith, Simple Noetherian rings (Cambridge University Press, London, 1985).

[6] N.V. Dung and P.F. Smith, 'On semi-artinian V-modules', J. Pure Appl. Algebra 82 (1992), 27-37.

[7] N.V. Dung, D.V. Huynh, P.F. Smith and R. Wisbauer, Extending modules (Pitman, London, 1994).

[8] C. Faith, Algebra II: Ring theory (Springer-Verlag, Berlin, Heidelberg, New York, 1976).

[9] K.R. Goodearl, Singular torsion and the splitting properties (Memoirs of the Amer. Math. Soc., No 124, 1972).

[10] D.V. Huynh and R. Wisbauer, 'A structure theorem for SI-modules', Glasgow Math. J. 34 (1992), 83-89.

[11] S.H. Mohamed and B.J. Müller, Continuous and discrete modules, London Math. Soc. Lecture Note Series 147 (Cambridge University Press, London, 1990).

[12] B.L. Osofsky, 'Non-quasi-continuous quotients of finitely generated quasi-continuous modules', in Ring Theory, Proceedings Denison Conference 1992 (World Scientific, Singapore, 1993), pp. 259-275.

[13] B.L. Osofsky and P.F. Smith, 'Cyclic modules whose quotients have complement submodules direct summands', J. Algebra 139 (1991), 342-354.

[14] S.T. Rizvi and M.F. Yousif, 'On continuous and singular modules', in Non-Commutative Ring Theory, Proceedings Conference Athens, OH (USA) 1989, Lecture Notes Math. 1448 (Springer-Verlag, Berlin, Heidelberg, New York, 1990), pp. 116-124.

[15] N.V. Sanh, 'On weakly SI-modules', Bull. Austral. Math. Soc. 49 (1994), 159-164.

[16] P.F. Smith, 'Decomposing modules into projectives and injectives', Pacific J. Math 76 (1978), 247-266.

[17] P.F. Smith, 'Rings characterized by their cyclic modules', Canad. J. Math. 31 (1979), 93-111.

[18] B. Stenström, Rings of quotients (Springer-Verlag, Berlin, Heidelberg, New York, 1975).

[19] R. Wisbauer, Foundations of module and ring theory (Gordon and Breach, Reading, 1991).

[20] M.F. Yousif, 'SI-modules', Math. J. Okayama Univ. 28 (1986), 133-146.

Institute of Mathematics

PO Box 631

Boho, Hanoi

Vietnam
Department of Mathematics

Hue University of Pedagogy

32 Le Loi St

Hue, Vietnam 